\title{
Grading Exams and Homework More Efficiently and Effectively
}

We have found that Gradescope software (www.Gradescope.com) significantly reduces grading time for assignments and exams. For example, it reduced the grading time for each grader from 8 to 6 hours for a two-hour heat transfer exam with 150 students. Moreover, Gradescope provides faster and more detailed feedback to students, eliminates the need to return graded exam or assignment papers, and provides statistics on performance on individual problems. Using Gradescope for group assignments has the added advantage that all group members can see the assignment grading and feedback. All grading is done online with Gradescope. Students upload a PDF file for assignments. For exams, the instructor uploads a blank copy of the exam and indicates the locations for the student name and each problem solution, and enters points for each problem. The instructor then scans students' exams in batches and uploads PDF files to Gradescope. Multiple graders can then grade assignments or exams simultaneously. When grading is complete, students view the feedback and grades online. No papers are returned to students, avoiding possible privacy issues. Students can also submit regrade requests online.

A big advantage of Gradescope is that the same problem can be graded for each student by clicking a button to move to the next student's solution. This makes grading faster than handling paper exams. Once a rubric with feedback is entered into Gradescope for a problem, it can be used for multiple students, whereas for paper exams, the same feedback must be written multiple times. Points are subtracted by selecting a button for that rubric (Figure 1), so that grading is more consistent. Gradescope then adds the points for each student. Gradescope is free for the first year of use by an instructor, and then costs $\$ 1 /$ student/course.

Tips for using Gradescope

- Grading is most efficient if student solutions are written directly on the exam pages.

- Make exams two-sided and use a high-speed scanner that scans both sides simultaneously.

- The first exam page should be distinctive to aid in identifying the start of a student's exam.

- Students should indicate if they use an additional solution page so all pages are graded. $\square$

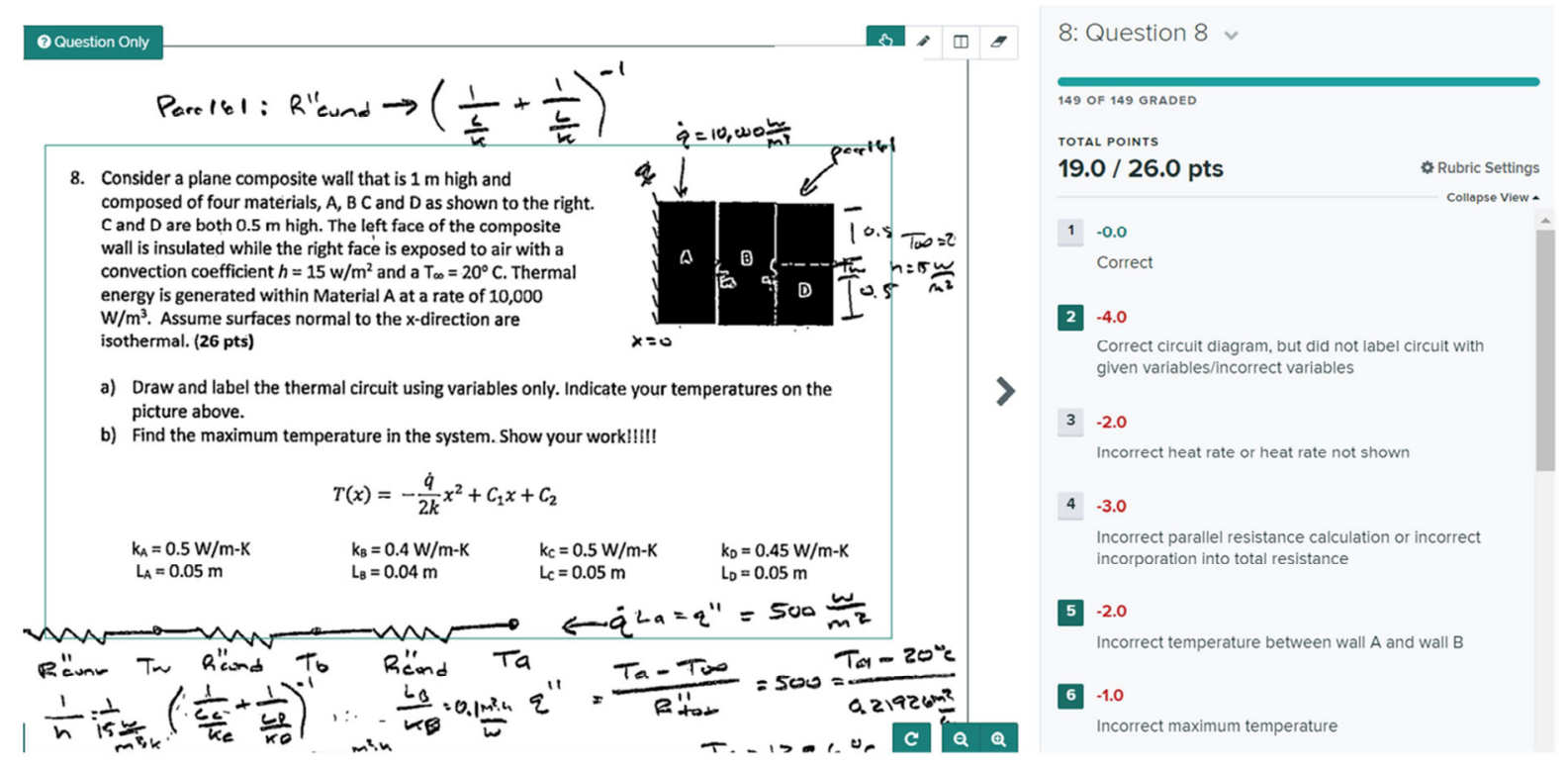

Figure 1. Example of student solution on Gradescope and the grading rubric.

— John L. Falconer and Janet deGrazia, University of Colorado Boulder, Boulder, CO

Teaching Tips are now peer reviewed. Submit 1 page Teaching Tip through journals.fcla.edu/cee, include Teaching Tip in the title, and specify Teaching Tip as the article type. 